\title{
Un testimonio del dios «Labbo» en una inscripción lusitana de Plasencia, Cáceres. $i \ll$ Labbo» también en Cabeço das Fráguas?
}

\author{
Eustaquio Sánchez Salor \\ Universidad de Extremadura \\ esanchez@unex.es \\ ORCID iD: https://orcid.org/0000-0001-9711-6349 \\ Julio Esteban Ortega \\ Universidad de Extremadura \\ jesteban@unex.es \\ ORCID iD: https://orcid.org/0000-0002-7628-8333
}

\section{A Testimony of God «Labbo» in a Lusitanian Inscription from Plasencia, Cáceres. «Labbo» also in Cabeço das Fraguas?}

Se ha venido defendiendo la identidad de Labbo como una divinidad de carácter local con culto restringido al territorio del Cabeço das Fráguas, donde hasta ahora se documentaba la única inscripción, que planteaba algunas dudas de lectura Laebo / Labbo. Presentamos aquí un ara votiva de Plasencia que demuestra con total seguridad la existencia de esta divinidad lusitana y la extensión de su culto hacia zonas más orientales del territorio lusitano. Su integración en una tríada de deidades nos lleva a comparar ambos textos y considerar en Cabeço das Fráguas dos series de nombres: seis de animales, por un lado, y seis de divinidades, por otro.

Palabras clave: Epigrafía; Lusitano; Divinidades Indígenas; Lingüística Indoeuropea.
The identity of Labbo has been defended as a local divinity, whose worship was restricted to the territory of Cabeço das Fráguas, the location of the only inscription documenting the name (variously read as Laebo or Labbo) that had been identified so far. We present here a votive altar from Plasencia that demonstrates with total certainty the existence of this Lusitanian divinity and the extension of its cult towards more eastern areas of the Lusitanian territory. Its integration into a triad of deities leads us to compare both texts and to consider two series of names in Cabeço das Fráguas: six of animals, on the one hand, and six of divinities, on the other.

Key words: Epigraphy; Lusitanian; Indigenous Divinities; Indo-European Linguistics.

Cómo citar este artículo / Citation: Sánchez Salor, Eustaquio y Esteban Ortega, Julio (2021): «Un testimonio del dios «Labbo» en una inscripción lusitana de Plasencia, Cáceres. ¿«Labbo» también en Cabeço das Fráguas?», Emerita 89 (1), pp. 105-126.

Copyright: (C) 2021 CSIC. Este es un artículo de acceso abierto distribuido bajo los términos de la licencia de uso y distribución Creative Commons Reconocimiento 4.0 Internacional (CC BY 4.0). 
Hace pocas fechas llegó a nuestro poder una fotografía de una inscripción romana procedente de Plasencia con un «enigmático texto» que parecía hacer alusión a una divinidad indígena, Labbo. Este teónimo se repite en la conocida inscripción rupestre del castro de Cabeço das Fráguas localizado en Quinta de São Domingos, al oeste de la freguesía portuguesa de Pousafoles do Bispo, Sabugal, Guarda (Rodrigues 1959, pp. 71-75). Sin embargo, el deterioro del texto de esta inscripción ha motivado no poca controversia, sobre todo en lo que se refiere a la lectura del teónimo, que ha venido restituyéndose como Laebo. Más recientemente Untermann, a quien siguen ya buena parte de los investigadores, cree que hay que leer Labbo (Untermann 1997, pp. 756-758). Quienes así piensan ven en Labbo una divinidad de culto local, reducido al ámbito del propio castro (Santos 2007, p. 83); la falta de otros testimonios así parecía aconsejarlo. No obstante, la existencia de un paralelo Laepo, testimoniado por tres veces en epígrafes cercanos a Cabeço das Fráguas, ha determinado que algunos estudiosos sigan inclinándose por la primera lectura y rechacen una posible relación entre Laebo y Labbo.

Presentamos aquí una inscripción inédita de Plasencia (Cáceres), en la que la lectura Labbo es indubitable. De esta manera la propuesta de Untermann cuenta ya con un paralelo que hasta ahora no tenía y nos ofrece la posibilidad de reinterpretar algunos detalles de la inscripción de Cabeço das Fráguas.

Aunque con caracteres latinos, la inscripción parece estar en lengua lusitana, al menos el componente teonímico; lo incompleto del texto impide confirmar si lo estaba en su totalidad. Y no es el primer caso documentado en la zona de Plasencia, pues se conoce otra ara con estas características hallada en la vecina localidad de Arroyomolinos de la Vera ${ }^{1}$, en la entrada de la comarca del mismo nombre. También está redactada en latín, con los teónimos en dativo y declinados siguiendo la morfología lusitana ${ }^{2}$.

\footnotetext{
${ }^{1}$ La editio princeps es obra de Beltrán (1975-1976, pp. 76-77); CILCC V, pp. 19-20, $\mathrm{n}^{\mathrm{o}} 1$.

${ }^{2}$ Los autores de este trabajo presentamos una nueva interpretación de esta inscripción, que se encuentra actualmente en fase de edición y que pronto verá la luz.
} 


\section{LA INSCRIPCIÓN INÉdITA DE PlaSENCIA}

Las noticias acerca de la aparición de esta inscripción en la capital del Jerte se la debemos a nuestro querido amigo José Antonio Pajuelo Jiménez ${ }^{3}$, quien nos informó de su ubicación en una casa adosada a la muralla que circunda el casco antiguo de la ciudad. Por las informaciones que nos han llegado a través de un miembro de la familia propietaria de la misma, sabemos que hasta hace pocas fechas el ara estaba partida pero se conservaban las dos mitades. Recientemente hemos tenido ocasión de visitar la mencionada vivienda y solo pudimos encontrar el fragmento correspondiente a la base. Ambos bloques estuvieron durante mucho tiempo en el piso superior de la casa, donde se puede ver el alzado de la muralla que en este tramo está bastante deteriorada. Probablemente nuestra inscripción pudo formar parte de este lienzo de muralla, de donde debió de ser extraída por los dueños de la vivienda en un momento que no podemos precisar. Recientemente se llevaron a cabo unas obras de consolidación de la misma y la parte superior desapareció. Sabemos que el ara tenía grabada en la cabecera una figura de animal, por lo que es muy probable que alguno de los operarios que realizaron la obra pudiera habérsela llevado como objeto de adorno. Sin embargo, no se puede descartar que fuera arrojada a la escombrera entre los cascotes de la obra sin que el autor se percatara de la verdadera importancia del bloque que desechaba. Puesto en contacto con uno de los albañiles que realizaron la obra intentamos averiguar la ubicación de la escombrera, no supo darnos referencias concretas. En cualquier caso, no perdemos la esperanza de encontrar el fragmento perdido que se nos antoja fundamental para comprender el texto de la inscripción.

Se trata de la parte inferior de un ara de granito correspondiente a la base y buena parte del fuste. El bloque está roto a bisel en la parte superior y el texto está incompleto. La base es muy irregular y de tosca elaboración, consistente en una protuberancia informe sin las características molduras de este tipo de monumentos (Fig. 1).

\footnotetext{
${ }^{3}$ Nuestro sincero agradecimiento al ilustre galeno placentino por mostrarnos esta nueva e interesante inscripción.
} 


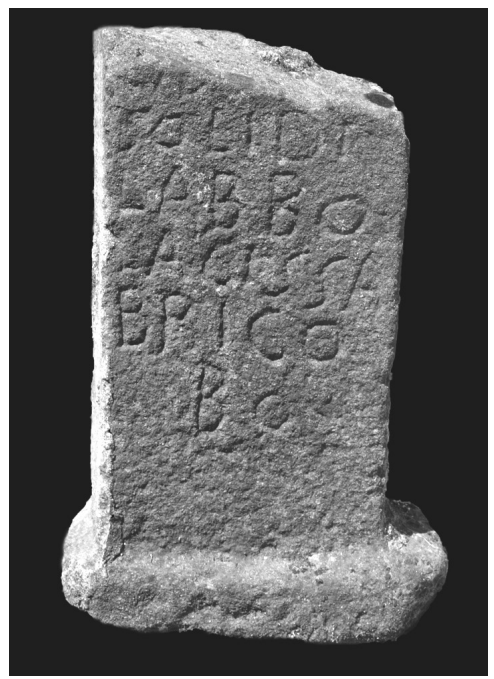

Fig. 1. Ara dedicada al dios Labbo en Plasencia (Cáceres).

Dimensiones: (55) x 37 x 16; letras 5-7.

\author{
$-----+?$ \\ [-]VB?+[-c.3-4-] \\ SOLIDI $\cdot$ \\ LABBO · \\ LACISSA- \\ 5 BRICO- \\ $\mathrm{BO}$
}

Traducción: «....a la diosa Solis, al dios Labo, a los dioses protectores de Lacissa».

La inscripción se desenvuelve en seis líneas de texto, la primera parcialmente afectada por la rotura. El estilo tosco de la talla y el descuidado grabado de las letras y la deficiente paginación sugieren un trabajo doméstico más que el resultado de una oficina especializada en la producción epigráfica. Las letras, muy toscas, desiguales y con trazos irregulares, son capitales muy rústicas y solo se observan signos de interpunción en punto al final de la tercera y cuarta líneas.

Línea 1: Solo se conservan algunos trazos de las letras iniciales. El primer grafo se ha borrado completamente y a continuación se aprecia un trazo ascendente inclinado a la derecha que pudiera corresponder a una V. Le sigue la 
parte inferior de una tercera letra que se asemeja bastante a las BB que aparecen en el texto. Quizás en esta línea haya que interpretar el antropónimo Lubaecus.

Línea 2: La S inicial está parcialmente borrada pero se lee sin dificultad. $\mathrm{La} \mathrm{O}$ es muy irregular.

Línea 3: Se lee perfectamente la palabra LABBO. La A parcialmente borrada; las $\mathrm{BB}$ muy irregulares y la $\mathrm{O}$, de menor tamaño, en forma oval más que redonda.

Línea 4: La primera letra está muy borrada, pero debe de tratarse de una L. La tercera puede ser C o G. Las SS aparecen algo tendidas con las astas curvas poco marcadas. La A final ha perdido parte del asta derecha.

Línea 5: La B inicial es más proporcionada. La $\mathrm{R}$ ha perdido la pata y la $\mathrm{I}$, al igual que las restantes aparecidas en el texto, es de menor tamaño. La cuarta letra puede ser $\mathrm{C}$ o $\mathrm{G}$ y la $\mathrm{O}$ final es irregular y de dimensiones más reducidas.

Línea 6: Comienza con una $\mathrm{B}$ también irregular y remata una $\mathrm{O}$, esta sí, redonda, más pequeña.

No es segura la existencia de una primera línea por encima de la que parcialmente aparece en el texto. Si fuera así habría que interpretar un devoto de nombre desconocido en nominativo, seguido de una segunda línea con el antropónimo de la filiación en genitivo, quizás Lubaeci, como señalábamos más arriba. En caso de no faltar ninguna línea el devoto sería el propio Lubaecus, que aparecería sin filiación y en nominativo.

Un nombre indígena como Lubaecus vendría acorde con el contexto epigráfico y la veneración de divinidades vernáculas tan poco frecuentes como las documentadas en el texto. Además, este nombre se repite en territorio caperense en otra inscripción de Santibáñez el Bajo (CILCC III, 1111). Lubaecus es un antropónimo básicamente lusitano pues sus testimonios epigráficos se concentran en esta provincia romana (Vallejo 2005, pp. 234-236), a excepción de un solo caso hallado entre los astures en la localidad leonesa de Santa Colomba de Somoza (Diego Santos 1986, p. 232). Los ejemplares extremeños se localizan principalmente al sur del Tajo, en las proximidades de Turgalium, como en Ibahernando (CILCC II, 544 y 577), con dos testimonios; Abertura (CILCC II, 437) y Torre de Santa María (CILCC I, 358). Una variante de este nombre es Libaecus, que encontramos en el cercano municipio de Plasenzuela (CILCC II, 648).

Vayamos ahora a SOLIDI LABBO LACISSABRICOBO ${ }^{4}$. Un primer acercamiento a la estructura de la inscripción parece llevarnos, efectivamen-

\footnotetext{
${ }^{4}$ Queremos dejar claro desde el principio que las propuestas se hacen desde la prudencia que es recomendable en estos casos y nunca desde una postura dogmática.
} 
te, al típico texto compuesto por el nombre del devoto, con o sin filiación, + teónimo + epíteto, sin fórmula votiva. Pero las terminaciones de dativo en los tres nombres parecen indicar que se trata de destinatarios, con toda probabilidad dioses. ¿Son tres dioses diferentes o uno solo con apelativos? Si tenemos en cuenta que Labbo y Lacissabricobo no pueden formar sintagma (nombre + adjetivo), porque uno es singular y otro plural, y tenemos también en cuenta que Labbo aparece solo en la inscripción de Cabeço, debemos concluir que se trata de tres dioses diferentes.

SOLIDI. Hemos de reconocer, como decíamos, que la primera impresión fue considerar Solidi como la filiación del dedicante y si bien la idea parece factible, ello supondría la aceptación de un nuevo antropónimo hasta ahora desconocido en la epigrafía peninsular y, que sepamos, la onomástica lusitana no documenta hasta la fecha ningún nombre con este mismo radical. Por todo ello nos planteamos la posibilidad de considerarlo como el dativo del nombre de una diosa; tenemos atestiguada una formación similar como MVNIDI en MVNIDI EBE/ROBRIGAE /TOVDOPALANDAIGAE AM/MAIA BOVTILA, en la cercana localidad de Talaván (CILCC I, 340); o MVNIDI IGAED(ITANAE o ITANORVM) / BA[E]VIA..., ya en la portuguesa Castelo Branco $(A E$ 1967, 142). Prósper (2002, pp. 187 ss.; cf. Prósper y Villar 2009, pp. 1-32.) pone en relación este teónimo con la raíz *men/mon, 'cabeza', 'monte', de manera que sería el dativo de un teónimo protector de la montaña.

Si aceptamos que Solidi es dativo como Munidi, estaríamos ante un dativo, cuyo nominativo sería Solis. En celta tenemos la diosa Sulis; es una diosa celta ctónica relacionada con las aguas subterráneas, cálidas y medicinales; en concreto era la diosa tutelar de las aguas termales de Bath (Inglaterra). Son muchas las ninfas o diosas menores que protegen los manantiales y corrientes de agua. Puede tratarse de la misma diosa, con vocalismo diferente. Pokorny (1959, s. u. Sal-) dice: «mhd. sol, sul, nhd. Sole 'salzhaltiges Wasser'» (en medio altomedieval alemán, tenemos sol, sul, en alto alemán moderno, Sole, 'riego de agua'). En la misma entrada, Sal-, muy frecuente en las lenguas indoeuropeas para designar conceptos relacionados con el agua, Pokorny recoge testimonios de sal-, sol- sul- en diferentes lenguas indoeuropeas. Salus, salutis en latín se encuentra con frecuencia en epigrafía para designar a una fuente. De manera que, si Munidi en Talaván es la diosa del monte, Solidi en Plasencia puede ser la diosa de la fuente o del río.

Algunos autores han interpretado a Sulis como una diosa solar. En nuestro caso podría tratarse de la diosa Sol. No hay que olvidar que en la mitología 
nórdica Sol es una diosa, conocida también como Sunna; la diosa del sol. De todas formas, nos inclinamos más por la relación con fuente o agua.

LABBO. En relación con Labbo, Prósper (2002, pp. 49-51), al analizar la conocida inscripción de Cabeço das Fraguas, advierte que con frecuencia se lee Laebo, que Untermann ha leído Labbo, y que tres inscripciones sobre altares de granito halladas en las laderas de Cabeço das Fráguas están dedicadas a Laepo, cuya lectura es indubitable. Supone que se trata de la misma deidad, cuyo nombre va en dativo, como destinataria del ara votiva. Señala también que hasta el momento su etimología es completamente oscura. Sí asegura que "parece claro que estamos ante una forma de dativo correspondiente a un sustantivo temático masculino o neutro cuya forma original era *laipo». Y añade que hay que excluir la etimología con sonora $/ \mathrm{b} / \mathrm{o} / \mathrm{bh} / \mathrm{y}$ naturalmente una posible con /w/; la conservación de étimos con la sorda /p/, que es la originaria, le hace pensar a esta autora que la inscripción no es celta, lengua que carece del fonema indoeuropeo /p/, sino lusitana. Y se atreve a asegurar que las formas Laebo y Laepo se refieren con toda probabilidad a la misma divinidad, que la identificación de estas dos formas, semántica y estructuralmente, es completamente impecable, y que la deidad Labbo es totalmente desconocida. En definitiva, insinúa que Labbo no es una buena lectura de Untermann, ya que es difícil poner esta forma en relación con Laebo o Laepo.

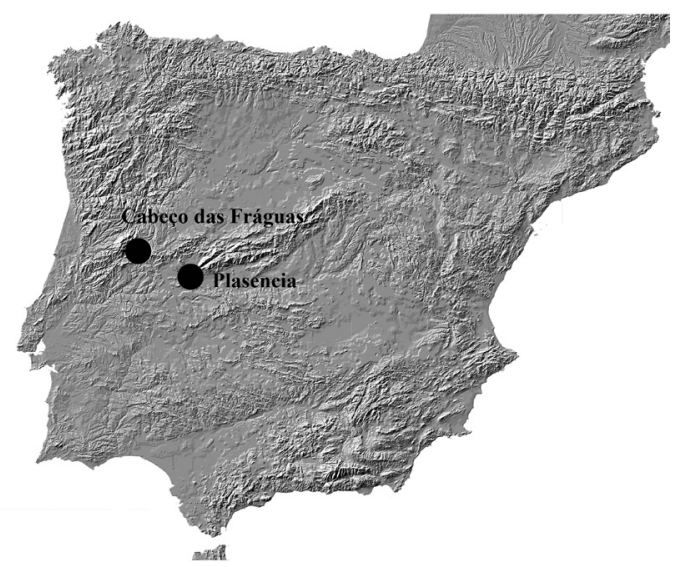

Fig. 2. Mapa de distribución de las aras de Labbo.

La lectura Labbo planteaba serias dudas puesto que no existían paralelos del nombre ni de la geminación de la $b$ en toda Lusitania. Pero es el caso que en 
la inscripción inédita de Plasencia, en la segunda línea que se conserva, la lectura es muy clara; se lee claramente Labbo. Además, recientemente apareció no muy lejos de aquí una inscripción muy escueta dedicada a una divinidad desconocida hasta la fecha, cuyo nombre, Vabbo, se acerca bastante al del epígrafe de Plasencia y confirma el fenómeno de la geminación. Se trata de un ara de granito, de talla muy tosca, hallada en la localidad de Villar del Rey, municipio cacereño situado al suroeste de la capital (Esteban Ortega 2017). Está partida en su parte inferior, aunque todo parece indicar que el texto está completo. Es muy breve, pues consta solamente del genérico deo seguido del teónimo también en dativo, sin dedicante ni formulario votivo, por lo que es muy posible que el ara estuviera destinada a un santuario particular de algún devoto.

Conviene, por tanto, explorar nuevas explicaciones, como señala Prósper, y lo haremos más adelante cuando comparemos esta inscripción de Plasencia con la de Cabeço das Fráguas.

LACISSABRICOBO. El tercer destinatario es Lacissabricobo. Este nombre es fácilmente analizable en tres componentes: Lac-; -ssa o-issa; y -briga.

El primer componente lac- está ampliamente atestiguado en la zona: Lacimurga en Navalvillar de Pela; Lacipea es una mansio cercana a Mérida, recogida en el Itinerario de Antonino como primera parada en el camino de Mérida a Zaragoza; Lacobriga en la costa sur de Portugal, actual Lagos del Algarbe; habría que comentar que la formación de Lacobriga es la misma que Lacissobriga, con la diferencia de que el topónimo de nuestra inscripción portaba ya, cuando se sumó el componente -briga, otro componente hispanocelta, -ssa-. Otros testimonios con la raíz lac- son Lacetani, pueblo ibero que habitaba en el Pirineo central. Lacipo en Málaga; también en este caso hay que comentar que el topónimo es portador del sufijo -ipo, que está en muchos topónimos e hidrónimos del sur de la península (Villar 2000, pp. 86-116), y que posiblemente sea oriental -de hecho, se encuentra en todo el territorio circunmediterráneo-, como lo es -ssa, de Larissa, según veremos. Otro testimonio es Laccuris, población oretana citada por Tolomeo. De Guimaraes es una inscripción dedicada a Genio Laquiniesi. Prósper (2002, p. 313) interpreta que Laquinie(n)si tiene que ver con la raíz id. *laku-, 'depresión', 'fosa', 'lago'.

En lo que se refiere al componente -(i)ssa, Elvira Gangutia (1998, pp. 106-107), hablando de Tartessos dice que la terminación -(e)ssós es llamativa por su notable arcaísmo. Se trata de la aplicación griega de un sufijo de procedencia indoeuropea que se encuentra en numerosos nombres anatolios en -(a)ssa con un valor 'pertinentivo' en luvita que sustituiría a un genitivo. 
Aunque el sufijo aparece con mayor densidad en Anatolia y la Hélade, puede encontrarse en Sicilia, sur de Italia y no es infrecuente fuera de esta área. Cabe pensar que en una época suficientemente arcaica para que el sufijo -(e)ssós de formación de topónimos e hidrónimos todavía estuviera 'vivo', griegos procedentes de las costas de Asia Menor en contacto con pueblos de lengua luvoide lo añadieron a temas indígenas de la península ibérica, procedimiento ampliamente utilizado en Asia Menor: hetitas y luvitas para crear ciertos topónimos o hidrónimos añadirían simplemente -ssa a un nombre, no necesariamente indoeuropeo.

De manera que -(i)ssa, según la propuesta que acabamos de ver, se añadiría a una raíz indígena, en este caso lac-. Y a su vez, a la forma resultante, Lacissa -formación similar a la de Larissa, la capital de Tesalia- se añadiría un segundo componente celta sobradamente conocido, -briga.

La terminación -bo es la de dativo de plural, conocida por lo demás en otras inscripciones de la zona ${ }^{5}$.

De manera que Lacissabricobo sería el dativo del plural de un nominativo del plural Lacissabrici, que podrían ser los dioses protectores del lugar, Lacissabrica o Lacissabriga.

\section{InSCRIPCIÓN DE CABEÇO dAS Fraguas}

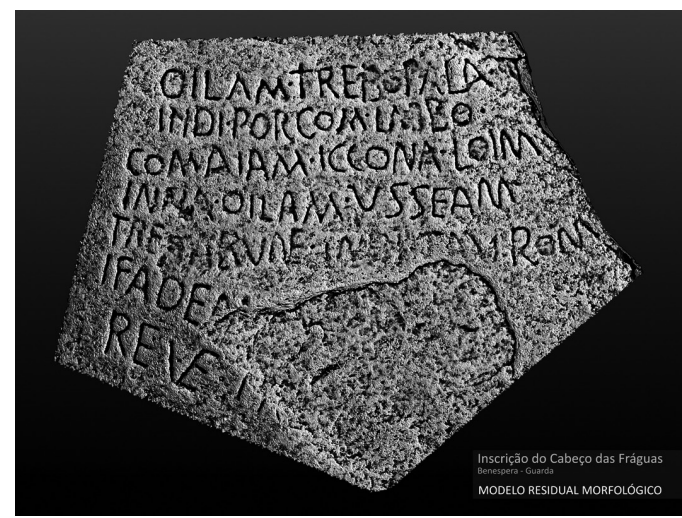

Fig. 3. Inscripción de Cabeço das Fráguas, Benespera-Guarda (MRM cedido por J. Cardim y H. Pires).

\footnotetext{
${ }^{5}$ Sobre los dativos del plural en -bo véase Marco Simón 1997, pp. 481-489.
} 
La lectura que se ha hecho de esta inscripción (Prósper 2002, p. 41, de Untermann 1997, pp. 756-757) es ${ }^{6}$ :

OILAM· TREBOPALA

INDI· PORCOM· LABBO.

COMAIAM· ICCONA· LOIM·

INNA· OILAM· VSSEAM.

TREBARUNE· INDI· TAVROM

IFADEM [---]

REVE $++[---]$

Esta inscripción ha sido estudiada por todos los que de una manera u otra se han acercado al mundo de los lusitanos. Entre los más recientes y más autorizados se encuentran Villar y Prósper. En buena medida coincidimos con ellos en la interpretación de la mayoría de las palabras. Pero hay puntos en los que nos atrevemos a proponer una nueva interpretación. Es ello lo que nos ha empujado a presentar nuestra opinión.

Villar distingue entre dos métodos a la hora de acercarse a la interpretación de las inscripciones en general y de esta en particular, a los que llama método combinatorio ${ }^{7}$ y método etimológico. El primero se refiere a la posición desde la que se contempla la inscripción como una estructura organizada en las que los constituyentes se explican a partir de la función de otros constituyentes del conjunto. El segundo se refiere exclusivamente a la fuerza probatoria de la doctrina fonética y morfológica que explica la evolución de las raíces y palabras indoeuropeas en las diferentes lenguas; evidentemente de las raíces y palabras que aparecen en la inscripción.

Nuestro punto de partida es precisamente el método combinatorio, entendiendo por tal el recurso de poner en relación los constituyentes nominales del epígrafe en la medida en que puedan tener sentido precisamente por formar parte de una estructura organizada.

${ }^{6}$ Debemos la cesión de este magnífico material gráfico a José Cardim Ribeiro y a Hugo Pires, a quienes agradecemos su gentileza. Este MRM se incluye en Cardim Ribeiro y Pires (en prensa).

7 Otra vez lo llama distribucional. Y, cuando el punto de referencia que pueda explicar determinadas formas está fuera de la propia inscripción, lo llama contrastivo; no es otra cosa que la existencia o no de paralelos fuera de la inscripción en cuestión. 
Independientemente de si se trata de una inscripción votiva, como afirman buena parte de los investigadores, o de una inuocatio como propone, para nosotros erróneamente, Cardim (2014, pp. 99-144), creemos que de la estructura compositiva del texto se desprende la existencia de nombres: de animales, por un lado, y de nombres propios de dioses, por otro.

\begin{tabular}{ll}
\multicolumn{1}{c}{ Nombres de animales } & Nombres de dioses \\
Oilam & Trebopala \\
Porcom & Labbo \\
Comaiam & Iccona \\
Loim & Inna \\
Oilam Usseam & Trebarune \\
Tauron Ifadem & Reue
\end{tabular}

En este esquema nos apartamos de las interpretaciones hasta ahora ofrecidas en un punto: siempre se ha leído Loiminna como una sola palabra e interpretado como epíteto de Iccona. Nosotros interpretamos Loim, por un lado, que sería un animal, e Inna por otro, que sería una diosa. Hay que dar una explicación a este respecto: en la propuesta que hacemos tenemos seis animales, o mejor, dos tríos de animales: dos ovejas, dos cerdos y dos bóvidos; y tenemos seis dioses, a cada uno de los cuales se dedica un animal.

Bien es cierto que en la inscripción de Arroyo de la Luz, dada a conocer por Gómez-Moreno (1949, p. 204), siguiendo a Hübner (1893, XLVI-XLVII) encontramos el nombre Loemina, que Villar y Pedrero (2001, p. 243) consideran como la única coincidencia entre Arroyo y Cabeço. Pero dado que nadie se atreve a lanzar una interpretación del Loemina de Arroyo; que es dudosa su lectura, ya que algunos proponen Goemina; y que se trata de una inscripción que no se conserva, sino que fue transmitida por Masdeu, este testimonio no tiene la fuerza suficiente como para exigir que el Loiminna de Cabeço sea una sola palabra. Lo que hay que hacer es interprerar el Loemina de Arroyo $^{8}$. Prósper (2002, p. 53) sostiene que el epíteto Loiminna de Cabeço es idéntico a la palabra Loemina de la inscripción de Arroyo de la Luz, Cáceres (Untermann 1997, p. 749). Añade que su etimología tiene que ver con

\footnotetext{
${ }^{8}$ Sin razón que lo apoye insinuamos que podría leerse Loemim, nombre en acusativo en -im, como otros del epígrafe.
} 
*loimos, que en latín da limus. Pero la verdad es que de Loiminna dice que es un epíteto y de Loemina dirá que es un teónimo (2002, p. 78): no son, pues, lo mismo.

De manera que en Cabeço nos encontramos probablemente con doble suouetaurilia. No es algo extraño. Virgilio dice en Aen. V, 97-98 que Eneas, en los funerales de su padre Anquises, caedit binas de more bidentis / totque sues, totidem nigrantis terga iunencos («sacrificó dos ovejas según la costumbre, dos cerdos, y dos novillos con el espaldar de color negro»). Y en el Louvre se conserva un relieve de doble suouetaurilia en época Julio-Claudia, probablemente durante el reinado de Calígula.

\section{Los animales}

Hay, en primer lugar, tres animales que corresponden a los tres que forman parte del sacrificio conocido como suouetaurilia: oveja, puerco, vaca9 .

OILAM. Se trata de un acusativo que ha sido identificado por los estudiosos con 'oveja' o 'cordero'. Ya Tovar (1967) lo interpretó como una forma derivada del indoeuropeo *owis, 'oveja'; concretamente de la forma *owi-la; de manera que para Tovar se trataría de un diminutivo y, por tanto, de un cordero o cordera. Prósper apunta que tanto el diminutivo lusitano procedente de *owi-la, como el también diminutivo latino, ouicula, pudieron en algún momento dejar de significar, sin duda que por desgaste, diminutivo, para referirse a la oveja en general.

PORCOM. No cabe duda de que es el acusativo de un nombre común indoeuropeo con el que se aludía el cerdo. La palabra indoeuropea era *porkos, que más que 'cerdo', significaba, según Prósper (2002, p. 49), 'lechón'. Aparece en otra inscripción lusitana.

COMAIAM. Creemos que acierta Prósper (2002, p. 53) al proponer, frente a las opiniones de Tovar y Untermann ${ }^{10}$, que, de acuerdo con las conocidas equivalencias entre $\mathrm{C}$ y $\mathrm{G}$, como grafías ambas de $\mathrm{K}$, Comaiam puede ser también interpretado como Gomaiam. Estaríamos entonces ante la raíz indo-

\footnotetext{
${ }^{9}$ Oveja a Júpiter, cerdo a Tellus y toro a Marte.

${ }^{10}$ Estos autores especulaban dubitativamente con una lectura Comalam, que tendría que ver con el alemán Hammel, en cuyo caso estaríamos ante un carnero castrado y sin cuernos.
} 
europea ${ }^{*}$ gem- $/ \mathrm{gom}^{-11}$. Esta autora recuerda que las Tablas Iguvinas testimonian un adjetivo Kumiaf, Gomia, 'grávidas', cuya etimología *gom-ians es indiscutible. Lo que sí discute es la identificación del animal. En definitiva, debe tratarse de una hembra preñada. Dado que ya tenemos la oveja y el cerdo, y se supone que estamos ante el sacrificio conocido como suouetaurilia, deberíamos concluir que con Comaiam estamos ante una vaca: joven y preñada, porque era con el sacrificio de una vaca primeriza con el que se lustraban los campos en determinadas circunstancias. A Tellus y Ceres se les ofrecía una vaca joven preñada.

Siguen después otros tres animales. Se repite la oveja; se repite un animal bóvido: antes hemos visto a una vaca joven preñada, ahora se trata de un toro. Y el otro es Loim, que, por paralelismo, debe ser 'cerdo'.

Veamos cada uno de ellos.

LOIM. Pokorny (1959, p. 652) recoge la raíz indoeuropea *lai- con el significado de 'gordo/a'. De ella trae testimonios del griego y del latín. En latín tenemos laridum, lardum ('tocino', 'carne de cerdo') que Pokorny hace remontar a la forma *laies-idom; lae-tus 'bien nutrido', 'exuberante'. De laetus dicen Ernout y Meillet (1967, s. u. laetus) que es un «adjectif de langue rustique» que significaba 'graso'. Y, en relación con su etimología no encuentran «aucun rapprochement net pour ce mot populaire à vocalisme a». No conocen la etimología, pero insisten en que es un término de origen rústico y popular. Creemos que es la raíz *lai-/loi- (las variantes ae/oe son sobradamente conocidas). Palomar Lapesa (1957, p. 133) señala que la $a$ indoeuropea en general se ha mantenido como $a$ en la antroponimia lusitana; pero aparece como - $u$-, como resultado de -o-, en el antropónimo Dubra de Odrinhas, Lisboa, (CIL II, 5019), que interpreta como *dhabhr-o-s; en céltico, sigue diciendo, la $a$ ha pasado a $o$ cuando precede o sigue una labial, y este es el caso de Dubra. No tenemos que ir muy lejos, en nuestras lenguas actuales, para encontrar una forma que quizás tenga que ver con la forma de la que estamos hablando; en francés tenemos la forma laie o laye que se refiere a la jabalina, hembra del jabalí, palabra de la que suelen decir los diccionarios etimológicos del francés que es de origen desconocido.

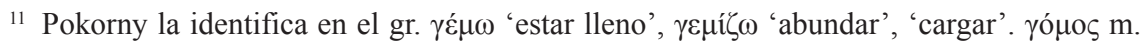
'carga', 'cargamento'; en umbro gomia, kumiaf, 'gravidās'; de ahí el latín gumia, -ae m. f. ('comilón', 'tripón'); y en otras lenguas indoeuropeas. 
En el caso de loim estaríamos, pues, ante el acusativo de un término como lois o lais, que aludiría a un cerdo cebado, gordo.

OILAM VSSEAM. Ya Tovar (1967, p. 244) interpretó oilam como 'oveja'. Prósper (2002, p. 47) dice que este sintagma hace desde luego alusión a una oveja. Y añade que Vsseam suele interpretarse como 'de un año'.

TAVRON IFADEM. Dice la misma autora que evidentemente es un toro. $\mathrm{Si}$ aceptamos la etimología de Tovar para ifadem (adjetivo deverbativo de *eibh-, *yebb, 'futuere'), se trataría de un toro entero, es decir, sin castrar.

\section{Los nombres propios de dioses}

Los nombres de los dioses que hasta ahora habían sido interpretados estarían, según Villar y Prósper, en dativo. Villar dedica un profundo análisis a la explicación de estos dativos. A propósito de los nombres en dativo de las inscripciones de Cabeço das Fráguas, Arroyo de la Luz y Lamas de Moledo Villar y Pedrero (2001, pp. 235-274) sostienen que en lusitano hay formas terminadas en $-a$ que pueden ser dativos:

Aparte de los citados Crougia y Iovea(i) tenemos: Arantia Ocela[e]ca, en Castelo Branco (RAP 11), Trebaronna, también en Castelo Branco (RAP 195), Lacipaea, en Mérida (Fita 1894), Domina Ataegina, en Bienvenida-Badajoz (Esteban 1982) y Frovida, en Braga (RAP 149). Y, por otra parte, el método combinatorio lleva a ver en Cabeço das Fráguas las formas Trebopala, Iccona, Loiminna y Labbo como dativos, al integrarse en sintagmas en los que a un acusativo sigue un caso que es un dativo cierto en las ocasiones en que nos es dado asegurarlo: 1) oilam usseam Trebarune; 2) taurom ifadem Reve Tre[. Sobre ese paralelo puede inferirse que tenemos la misma estructura sintáctica en los tres sintagmas por lo demás paralelos: 3) oilam Trebopala; 4) porcom Laebo; 5) comaiam Iccona Loiminna.

Con nuestra interpretación, se trataría, no de cinco sintagmas con la misma estructura sintáctica, sino de seis, ya que el quinto de Villar-Pedrero consideramos que pueden ser dos sintagmas: comaiam Iccona y loim Inna.

TREBOPALA. Prósper deja sentado desde el principio que es un compuesto, cuyo primer miembro es *trebo-, 'poblado', 'habitación'. Y discute largamente las diferentes propuestas que se han hecho para el segundo elemento -pala. Una de esas propuestas es la que ha puesto en relación este 
componente con la palabra alpina pala, 'monte', 'laja', que ha dado pie a comparaciones con el ligur, mediterráneo y lepóntico. Esta estudiosa no está convencida de que esta sea la solución correcta, ya que ella prefiere quedarse en el ámbito lusitano. Otra propuesta es la de Maggi (1983, pp. 53-60), que ha equiparado a Trebopala con la diosa védica Vispala; rechaza también Prósper (2002, p. 45) esta propuesta, porque Trebopala es probablemente «un híbrido y como tal no puede tener correspondencias directas» con diosas de otras culturas. En definitiva, esta autora considera que el teónimo se debe explicar «en términos internos, no ligures, etruscos o lepónticos» (ibíd.); y es que en la península tenemos multitud de topónimos con la raíz pal-/pel-, que significa 'fluir'; es una raíz formante de hidrónimos, de manera que el significado de este segundo elemento del teónimo es hidronímico. Termina proponiendo que significa 'la charca o arroyo del pueblo'. De manera que para Prósper, lo que se dice en la primera línea del epígrafe es «una oveja a la charca del poblado». Hemos de suponer que se trata de la charca del poblado divinizada.

Pensamos, sin entrar en profundos detalles etimológicos internos, que en Trebopala estamos ante una divinidad protectora de la casa o del poblado. En definitiva, protectora del pueblo protagonista de la inscripción. Esta es la conclusión a la que vamos a llegar cuando hagamos el definitivo análisis en conjunto del epígrafe, basándonos en las relaciones internas entre los constituyentes del mismo. Si esto es así, pala debe ser un constituyente (alpino o hispano, lepóntico o lusitano), que pudo adquirir el significado de 'protectora'. Hay que tener en cuenta que las palabras, cuando se crean, se creen en la lengua que se creen, tienen un significado muy general con aplicaciones en el plano de la dimensión física y en el plano de la abstracción. Un ejemplo: la preposición pro, en el plano de la dimensión física, significa 'delante de' si se trata del plano espacial, y 'antes de', si del temporal; pero en el plano de la abstracción significa 'en defensa de". Pues bien, pala, en el plano de la dimensión física, puede significar 'roca escarpada vertical', que está delante, si aceptamos la propuesta alpina; o puede significar 'corriente que fluye', que rodea, si aceptamos la propuesta hispana. Pero en ambos casos, en el plano de la abstracción, puede fácilmente terminar significando 'protección'. De manera que creemos que Trebopala es la diosa local protectora del lugar y del pueblo del lugar. 
$\angle A B B O$. La primera cuestión que plantea este nombre es la de la lectura ${ }^{12}$. Generalmente se ha leído Laebo; Untermann, sin embargo, leyó Labbo. En favor de Laebo se ha aducido la existencia de tres inscripciones sobre altares de granito halladas en una finca de Pousafoles (Sabugal, Guarda), prácticamente en la ladera de Cabeço das Fráguas; las tres están dedicadas a un dios Laepo $^{13}$. Basándose en la probabilidad de que Laepo y Laebo «se refieran a la misma deidad, podemos concluir», dice Prósper (1999, p. 169 y 2002, p. 51), «que Laebo debe ser el dativo de un sustantivo temático *laipo». En favor de Labbo está el hecho del testimonio de la inscripción de Plasencia inédita que presentamos también en este trabajo, en la que se lee claramente Labbo y en la que ese Labbo es, con toda probabilidad, el dativo de un sustantivo temático con el que se designaba a una divinidad.

¿Hay posibilidad de aceptar la lectura Labbo en Cabeço sin que ello suponga, como asegura Prósper, que se «desvincularía» la relación de la inscripción de Cabeço con las otras inscripciones de la zona en las que la deidad es Laepo? La conclusión implícita de esta autora es que, si queremos que el teónimo de Cabeço tenga relación con el Laepo de las otras tres inscripciones, hay que leer Laebo, y no Labbo, en Cabeço. Pero también se puede pensar que Laepo y Labbo no tienen nada que ver entre sí. Labbo no es ya una divinidad desconocida. Con la inscripción inédita de Plasencia, que aquí publicamos, esta divinidad Labbo deja de serlo. De manera que hay que explicar Labbo independientemente de Laepo. Para Labbo podemos pensar en la raíz *labh-, de la que dice Pokorny $(1959$, p. 652) que significa 'tomar', coger” y que está representada en ai. lábhatē, lámbhatē, rábhatē (perf. lalābha) 'se apodera', lābha- m. 'ganancia', 'victoria', 'beneficio'; gr.

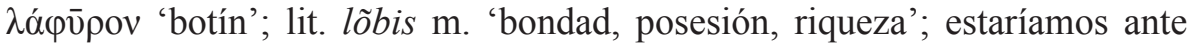
una raíz que puede utilizarse para designar a una divinidad ctónica, cuya función es la de generar riqueza y abundancia. Hay otra raíz indoeuropea analizada por Pokorny que podría estar también, como alternativa, detrás de Labbo. Se trata de *lab-/ lap(h)-, que significaría 'sorber', 'lamer' (cf.

12 Sobre la lectura del teónimo véase el reciente trabajo de López y Vallejo 2018, pp. 251-265, donde se analizan los problemas de lectura de la inscripción del Cabeço das Fráguas a partir de la interpretación de los autores que trataron sobre ella, con especial atención a la divinidad Labbo.

${ }^{13}$ Vasconcelos 1913, pp. 618-620; Curado 1984, pp. 8-12; Osório 2002, pp. 4-9 y 2006, pp. $137-138, \mathrm{n}^{\circ} 9$. 


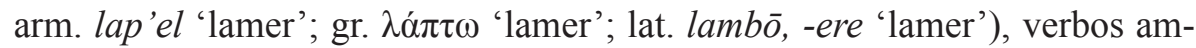
bos que aluden a la actividad típica de una cría que se alimenta de una madre; también en este caso podríamos estar ante una raíz que se utilizaría para designar a una divinidad ctónica, cuya función es la de generar riqueza y abundancia.

De manera que el cerdo de la inscripción de Cabeço sería sacrificado a una deidad ctónica, Labbus, relacionada con el alimento y la vida.

ICCONA. Se acepta generalmente la propuesta de interpretación que hizo Maggi (1983, pp. 53-60), quien la identificó con la diosa Epona.

Parece que hay consenso entre los estudiosos a la hora de identificar a Iccona/Epona con la gran diosa madre de todas las religiones orientales e indoeuropeas (Prósper 2002, pp. 51-53).

De manera que, junto a la diosa protectora del lugar, y junto a un dios ctónico que alimenta, tenemos, en la primera tríada del epígrafe, a la diosa madre. Y a ella se ofrece una ternera preñada.

Siguen otros tres dativos de teónimos. Son:

INNA. Acabamos de ver que la tercera diosa de la primera tríada del epígrafe es Iccona, considerada como diosa madre. Pues bien, la primera diosa de la segunda tríada del epígrafe es también la diosa madre, pero con otro nombre: Inna. Este es uno de los nombres de la diosa madre mesopotámica. Otros nombres de esta misma diosa eran Inanna, Istar, Astoret. Las diferentes manifestaciones de la diosa madre conservan siempre elementos procedentes de la diosa madre oriental. Prósper (2002, p. 52), citando a Meid (1991, p. 42 , n. 15), dice que este autor observa que Epona es idéntica a la "gran madre" o Matrona, llamada también Rigani en la Galia, *Riantona $>$ Rhiannon en el Mabinogion (colección de historias galesas), y Macha en la mitología irlandesa. Son madres de procedencia oriental.

TREBARUNE. El análisis e interpretación de Trebarune ha de ser el mismo que el que ya hicimos de Trebopala. Es un compuesto, cuyo primer elemento es *trebo-, 'poblado, habitación'. Del segundo, identificado por Prósper como *arunis, dice esta autora que, de la misma forma que pala, tiene también significado hidronímico. Y propone que sea interpretado como 'arroyo del pueblo'.

Con el mismo argumento que utilizamos en el caso de Trebopala, podemos también decir aquí que estamos ante la diosa protectora del lugar y del pueblo del lugar. 
REVE. Dos propuestas se han hecho a la hora de interpretar esta forma: una que pone en relación este teónimo con la raíz *reuz-, $r \bar{u}$-, que es la que ha dado en latín rus. Otra que lo pone en relación con la raíz *reu-, 'moverse', 'fluir', que daría hidrónimos. Tovar (1967, p. 261) propuso la primera; Villar (2003, p. 274) y Prósper (2009, p. 205) se inclinan por la segunda. Cualquiera de las dos etimologías propuestas puede explicar la interpretación de que se trata de un dios relacionado con la fertilidad y la abundancia. Si la etimología es *reuz-, rū-, que es la que ha dado en latín rus, no cabe duda de que estamos con un significado en relación con la tierra como fuente de productos saludables y de estado placentero. Si la raíz es *reu-, 'moverse', 'fluir', hay que pensar que esta raíz puede terminar por significar no el río en sí mismo, sino también las riberas y veras del río que son terrenos abundantes y fructíferos; placenteros también ${ }^{14}$.

En cuanto a la interpretación religiosa de Reve, Olivares (2000, pp. 208209) considera que la vinculación de esta divinidad con las montañas y las corrientes fluviales no vienen sino a confirmar su carácter de "dios supremo, celeste y controlador de los fenómenos atmosféricos". Esta identificación de Reve con la divinidad celestial es defendida también por Witczak (1999) y Mánczak (2006).

La ventaja de la interpretación que damos es que estructuralmente la inscripción queda organizada así: hay dos tríos de animales, dos ovejas, dos cerdos y dos bóvidos; con la particularidad de que los tres primeros parecen ser animales jóvenes, según las interpretaciones de los autores que han estudiado la inscripción: de oilam, como hemos visto, dicen Tovar y Prósper que se trataría de un cordero o cordera; de hecho, cuando se quiere indicar que se trata de una oveja y no de un cordero se añade el adjetivo usseam; de porcom dice Prósper, que, más que cerdo, sería un lechón; y de comaiam, hemos apuntado que se trataría de una novilla preñada primeriza. De los otros animales, parece que oilam usseam es una oveja de un año, según Prósper; ya hemos dicho que, si se añade el adjetivo usseam, debe ser para distinguirla de la simple oilam; taurom es un toro entero; y loim sería un cerdo cebado. En el caso de la inscripción de Cabeço, el cerdo se ofrece a Labbo, un lechón, y a Inna, un cerdo cebado. La oveja se ofrece a Trebarune, una cordera o

\footnotetext{
${ }^{14}$ Véase también Búa Carballo 2000.
} 
cordero, y a Trebarune, una oveja de un año. Y el bóvido es ofrecido a Icco$n a$, una novilla preñada, y Reve, un toro entero.

\section{BiBLIOGRAFÍA}

Albertos Firmat, M. ${ }^{a}$ L. (1985): «La onomástica personal indígena del Noroeste peninsular (astures y galaicos)», en De Hoz, J. (ed.), Actas del III Coloquio sobre Lenguas y Culturas Paleohispánicas, Salamanca, pp. 255-310.

Beltrán Lloris, M. (1975-1976): «Aportaciones a la epigrafía y arqueología romanas de Cáceres», Caesaraugusta 39-40, pp. 19-111.

Búa Carballo, J. C. (2000): Estudio lingüístico de la teonimia lusitano-gallega, Tesis Doctoral, Universidad de Salamanca.

Cardim Ribeiro, J. (2014): «Damos-te esta ovelha, ó Trebopala!” A invocatio lusitana de Cabeço das Fráguas», Conimbriga 53, pp. 99-144.

Cardim Ribeiro, J. y Pires, H. (en prensa): «Da fixação textual das inscrições lusitanas de Lamas de Moledo, Cabeço das Fráguas e Arronches: O contributo do "Modelo de Resíduo Morfológico" (MRM), seus resultados e principais consequências interpretativas», en Actas del XIII Coloquio Internacional de Lenguas y Culturas Prerromanas de la Península Ibérica, [Acta Palaeohispanica XIII = Palaeohispanica 20], Zaragoza.

CILCC I: Esteban Ortega, J. (2007): Corpus de Inscripciones latinas de Cáceres I. Norba, Cáceres.

CILCC II: Esteban Ortega, J. (2012): Corpus de Inscripciones latinas de Cáceres II. Turgalium, Cáceres.

CILCC III: Esteban Ortega,J. (2013): Corpus de Inscripciones latinas de Cáceres III. Capera, Cáceres.

CILCC V: Esteban Ortega, J. (2019): Corpus de Inscripciones latinas de Cáceres V. Augustobriga, Cáceres.

Curado, F. P. (1984): «Aras a Laepus procedentes de Pousafoles, Sabugal», Ficheiro Epigráfico 7 (28), pp. 8-12.

Curado, F. P. (1985): «Ara a Vortiaecius», Ficheiro Epigráfico (anexo de Conimbriga) 13, n. 57.

Diego Santos, F. (1986): Epigrafia romana de la provincia de León, León.

Durante, M. (1978): «I dialetti medio-italici», en Prosdocimi, A. L. (ed.), Popoli e civiltà dell'Italia antica, VI: lingue e dialetti, Roma, pp. 789-824.

Encarnação, J. de (1975): Divinidades indígenas sob o dominio romano en Portugal, Lisboa.

Ernout, A. y Meillet, A. (1967): Dictionnaire étymologique de la langue latine, París. 
Esteban Ortega, J. (1984): «Epígrafe sobre la diosa Ataecina en Bienvenida (Badajoz)», Actas de las II Jornadas de Metodología y Didáctica de la Historia. Historia Antigua (diciembre de 1981, Cáceres), Cáceres, pp. 21-26.

Esteban Ortega, J. (2017): «Inscripciones romanas inéditas del Museo de Cáceres», en González Bornay, M. (coord.), Arqueología urbana en Cáceres, II. Investigaciones e intervenciones recientes en la ciudad de Cáceres y su entorno: Actas de las II Jornadas de Arqueología del Museo de Cáceres, Cáceres, pp. 229-236.

Fita, F. (1894): «Excursiones Epigráficas», BRAH 25, pp. 94-95.

Gangutia Elícegui, E. (1998): La península ibérica en los autores griegos: de Homero a Platón, en Mangas, J. y Plácido, D. (eds.), Testimonia Hispaniae Antiqua II $A$, Madrid.

Garcia, J. M. (1991): Religiões antigas de Portugal. Aditamentos e observações ás Religiões da Lusitania de J. Leite de Vasconcelos, Lisboa.

Gómez Moreno, M. (1949): Misceláneas. Historia-Arte-Arqueología, Madrid.

Haba Quirós, S. (1990): «La divinidad Vortiacio: Nuevos testimonios epigráficos hallados en la provincia de Cáceres», Alcántara 19, pp. 121-132.

Hübner, E. (1893): Monumenta linguae Ibericae, Berlín.

López Fernández, A. y Vallejo Ruiz, J. M. (2018): «Laebo vs. Labbo: Precisiones sobre la lectura de la inscripción lusitana del Cabeço das Fráguas (Benespera, Guarda)», en Vallejo, J. M., Igartua, I. y García, C. (coord.), Studia philologica et diachronica in honorem Joaquín Gorrochategui: indoeuropaea et palaeohispanica, Bilbao, pp. 251-265.

Maggi, D. (1983): «Sui teonimi Trebopala e Iccona nell'inscrizione del Cabeço das Fráguas», Problemi di lingua e cultura nel campo indoeuropeo, Pisa, pp. 53-60.

Manczak, W. (2006): «Lusitanien REVE = Latin JOVI», Lingua Posnaniensis 48, pp. 63-65.

Marco Simón, F. (1997): «Deis Equenu(bo)», en Beltrán, F. y Villar, F., Pueblos, lenguas y escrituras en la Hispania prerromana: Actas de VII Coloquio sobre Lenguas y Culturas Paleohispánicas, Zaragoza, 12 a 15 de marzo de 1997, Zaragoza, pp. 481-490.

Meid, W. (1991): Aspekte der germanischen und keltischen Religion im Zeugnis der Sprache, Innsbruck, Institut für Sprachwissenschaft der Universität.

Monteiro, J. A. (1942): «Ara e cipo miliario inéditos», Novidades, 30 de agosto de 1942 y 6 de novembre de 1942.

Olivares, J. C. (2000): «Los dioses soberanos y los ríos en la religión indígena de la Hispania indoeuropea», Gerión 18, pp. 191-212.

Osório da Silva, M. (2002): «Duas aras da Quinta de S. Domingos (Pousafoles do Bispo, Sabugal)», Ficheiro Epigráfico 69 (310), pp. 4-9.

Osório da Silva, M. (2006): O Povoamento Romano do Alto Côa, Guarda. 
Palomar Lapesa, M. (1957): La onomástica personal pre-latina de la antigua Lusitania: estudio lingüístico, Salamanca.

Pokorny, J. (1959): Indogermanisches etymologisches Wörterbuch, Berna.

Prósper, B. M. ${ }^{\text {a }}$ (1999): «The inscription of Cabeço das Fráguas revisited. Lusitanian and Alteuropäisch Populations in the West of the Iberian Peninsula», Transactions of the Philological Society 97 (2), pp. 151-183.

Prósper, B. M. (2002): Lenguas y religiones prerromanas del Occidente de la Península Ibérica, Salamanca.

Prósper, B. M. (2009): «Reve Anabaraeco, divinidad acuática de Las Burgas (Orense)», Palaeohispanica 9, pp. 203-214.

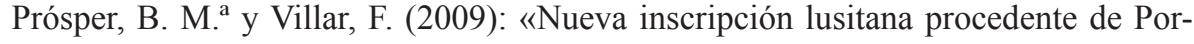
talegre», Emerita 77 (1), pp. 1-32.

$R A P$ : véase Garcia 1991.

Rodrigues, A. V. (1959): «Inscrição tipo 'Porcom' e aras anepígrafes do Cabeço das Fráguas (Guarda)», Humanitas 11-12, pp. 71-75.

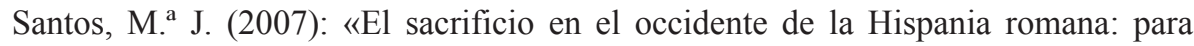
un nuevo análisis de los ritos de tradición indoeuropea», Palaeohispanica 7, pp. $175-217$.

Tovar, A. (1953): «Sustratos hispánicos y la inflexión románica en relación con infección céltica», en Badía, A. et al. (eds.), Actas del VII Congreso Internacional de Lingüistica Románica: Universidad de Barcelona, 7-10 abril de 1953, Barcelona, pp. 387-399.

Tovar, A. (1967): «L'inscription du Cabeço das Fraguas et la langue des lusitaniens”, Études Celtiques 11, pp. 237-268 (= «La inscripción de Cabeço das Fraguas y la lengua de los lusitanos», en De Hoz, J. (ed.), Actas del III Coloquio sobre Lenguas y Culturas Paleohispánicas, Salamanca, 1985, pp. 227-254).

Untermann, J. (1975): Monumenta Linguarum Hispanicarum I. Die Münzlegenden, Wiesbaden.

Untermann, J. (1997): «Die lusitanischen Inschriften», Monumenta Linguarum Hispanicarum. Band IV. Die tartessischen, keltiberischen und lusitanischen Inschriffen, Wiesbaden, pp. 756-758.

Vallejo Ruiz, J. M. (2005): Antroponimia indígena de la Lusitania romana, Vitoria.

Vallejo Ruiz, J. M. (2013): «Hacia una definición del lusitano», Acta Palaeohispanica XI = Palaeohispanica 13, pp. 273-291.

Vasconcelos, J. L. (1913): Religiões da Lusitania, vol. III, Lisboa.

Villar, F. (1986): «El dativo latino epigráfico en ā», Emerita 54, pp. 45-62.

Villar, F. (2000): Indoeuropeos y no indoeuropeos en la Hispania prerromana, Salamanca, pp. 86-116.

Villar, F. y Pedrero, R. (2001): «Arroyo de la Luz III», Palaeohispanica 1, pp. 235-274. 
Villar, F. y Prósper, B. M. ${ }^{\text {a }}$ (2003): «Nuevo epígrafe votivo dedicado a la divinidad Reve en la Coruña», Palaeohispanica 3, pp. 271-282.

Witczak, K. T. (1999): «On the Indo-European Origin of Two Lusitanian Theonyms (laebo and reve)», Emerita 67 (1), pp. 65-73.

Fecha de recepción de la primera versión del artículo: 08/07/2020

Fecha de aceptación: 18/08/2020

Fecha de recepción de la versión definitiva: 03/10/2020 